\title{
Demonstration of Physiological Barrier Between Pulpal Odontoblasts and its Perturbation Following Routine Restorative Procedures: A Horseradish Peroxidase Tracing Study in the Rat
}

\author{
D.F. TURNER, C.F. MARFURT', and C. SATTELBERG
}

Department of Oral Biology, The University of Michigan Dental School, Ann Arbor, Michigan 48109-1078; and 'Department of Anatomy, Indiana University School of Medicine, Northwest Center for Medical Education, Gary, Indiana 46408

\begin{abstract}
Vascular injection of the macromolecular tracer, horseradish peroxidase (HRP), was used to study the permeability of the odontoblast cell layer in developing and mature rat molar teeth, and to investigate the effect of cavity preparations on the permeability of this epithelioid cell layer in adult animals. HRP injected into the vascular system of normal animals 28 days of age and older was localized histochemically (from 5 to $90 \mathrm{~min}$ after injection) throughout the extracellular spaces of the maxillary dental pulps; however, the tracer did not penetrate beyond the tight junctions at the apical region of the odontoblast cell layer, and was absent from the predentin and dentin. In contrast, $H R P$ injected into very young neonatal animals (e.g., day 3) resulted in free passage of HRP between odontoblasts and into the overlying predentin and dentin. When Class $V$ cavities had been prepared in adult maxillary molars after HRP was injected into the blood stream, HRP reaction product penetrated the predentin and dentin immediately beneath the cavity preparation; however, adjacent, untraumatized areas of predentin and dentin in the operated teeth were devoid of reaction product. These results provide evidence that: (I) a physiological barrier develops between the distal segments of odontoblast cell bodies in normal rat molar teeth between days 15 and 28 of postnatal life, and this barrier prevents the passage of macromolecules from the pulp into the predentin and dentin; and (2) this barrier is perturbed following routine restorative procedures in adult animals.
\end{abstract}

\section{J Dent Res 68(8):1262-1268, August, 1989}

\section{Introduction.}

The odontoblast is a unique cell that functionally appears to be a hybrid between a connective tissue cell-highly specialized for the synthesis and secretion of the organic components of dentin-and an epithelial cell, functioning as a covering or liner for the dental pulp. During dentinogenesis, the odontoblast cell layer acts as a semi-permeable barrier between the extracellular compartments of the pulp and predentin, and selectively regulates the passage of molecules, ions, and water into the predentin, in order to create the proper ionic environment for calcification. To perform this function, specialized junctional barriers exist between the odontoblasts. Three basic types of intercellular junctions (i.e., gap junctions, desmosomes, and tight junctions) have been described in a variety of species, including humans (Arwill, 1967, 1968; Frank, 1968; Koling et al., 1981; Koling and Rask-Andersen, 1984a,b; Calle, 1985), monkeys (Turner, 1982), cats (Holland, 1975, 1976; Sasaki et al., 1982b; Bishop, 1987), rats (Jessen, 1967; Reith, 1968; Takuma and Nagai, 1971; Tanaka, 1980; Sasaki et al., 1982a; Iguchi et al., 1984), and mice (Garant et al., 1968). Most recent studies of odontoblast junctional morphology and

Received for publication November 14,1988

Accepted for publication March 28, 1989

D.F.T. was supported in part by N.I.H. Grant DE 06585 , and C.F.M. was supported in part by N.I.H. Grant EY 05717 and 507RR5371, awarded by The Biomedical Research Support Program of N.I.H. permeability have focused on the tight junction, and there is evidence to suggest that tight junctions contribute in an important way to (although they may not necessarily be the sole determinants of) this barrier function (e.g., Bishop, 1985). Unfortunately, the precise nature of the odontoblast tight junction has been only partly elucidated, and efforts to examine them by use of electron microscopy (Jessen, 1967; Garant et al., 1968; Reith, 1968; Frank, 1968; Takuma and Nagai, 1971; Holland, 1975; Bishop, 1987), freeze fracture (Koling et al, 1981; Koling and Rask-Andersen, 1984a,b; Sasaki et al., 1982b; Iguchi et al., 1984; Calle, 1985), and intercellular tracer technics (Holland, 1976; Tanaka, 1980; Sasaki et al., 1982a; Bishop, 1985) have so far yielded equivocal results. On the basis of these prior investigations, it has been variably concluded that tight junctions between odontoblasts are either absent (Koling et al., 1981), "macular", or discontinuous (Frank, 1968; Reith, 1968; Sasaki et al., 1982a,b; Iguchi et al., 1984), or "zonular", forming complete rings about the apices of the cells (Calle, 1985; Bishop, 1985). The reasons for the contrasting nature of these observations are unclear, but may include differences in the methodologies used or species examined, as well as differences in the developmental stage of the particular tooth under investigation.

The normal structure and function of the odontoblast cell layer may be adversely affected by a variety of pathological processes, including dental caries, attrition, and abrasion, and by the restorative procedures and materials used to treat them. The acute histological changes that take place in these circumstances include a loss of integrity of the odontoblast layer and aspiration of the odontoblast cell bodies into the dentinal tubules (Brännström, 1963; Stewart, 1965; Eda and Saito, 1978). The displacement of the odontoblasts is thought to result both from the outward flow of dentinal fluid at the surface of the exposed dentin, and from the mobilization of capillary forces (Johnson et al., 1973). Under these conditions, it seems likely that the tight junctions between adjacent odontoblasts would be stressed or broken, thereby compromising the epithelioid function of the odontoblast cell layer and increasing the potential for entry into the dental pulp of micro-organisms and their toxins. Unfortunately, our understanding of the shortterm mechanisms by which odontoblasts respond to dentin trauma and restorations remains rudimentary.

The current investigation was undertaken in an effort to: (1) determine whether functional intercellular barriers exist between odontoblasts in mature, fully erupted teeth and, if so, to determine the approximate age at which these barriers first appear, and (2) determine whether the normal barrier function of the adult epithelioid odontoblast cell layer is altered following routine restorative procedures. To study these questions, we have investigated the ability of pre-eruptive-as well as mature-odontoblasts to prevent passage of the enzyme tracer, horseradish peroxidase (HRP, mw 43,000), from the dental pulp to the predentin and dentin in normal teeth. We have contrasted these findings with observations on the permeability 
of the odontoblast cell layer to HRP, following Class V cavity preparations. The results of these studies have been presented previously in abstract form (Sattelberg and Turner, 1984, 1985).

\section{Materials and methods.}

We used a total of 22 adult Sprague-Dawley rats (each weighing from 200 to $250 \mathrm{~g}$ ) and 20 neonatal animals (ages from 3 to 28 days). All animals were carefully examined, and only animals with healthy dentition, free of obvious dental pathology, were selected for use. Each animal was anesthetized with an intraperitoneal dose of pentobarbital $(30 \mathrm{mg} / \mathrm{kg}$ body weight).

We used the neonates and 16 of the adult animals to study the permeability of the odontoblast cell layer in developing and fully erupted molar teeth, and the remaining six adult animals to investigate whether there was altered permeability of the odontoblast layer following Class V cavity preparations. In each adult rat in the first group, we made a midline incision on the ventral surface of the neck from the hyoid bone to the jugular notch, and gently separated the underlying strap muscles to expose the external carotid artery. We then carefully inserted a fine-tipped glass micropipette through the wall of the artery, and slowly injected from 0.2 to $0.4 \mathrm{~mL}$ of 1 to $5 \%$ HRP (Sigma type VI) in saline into the lumen of the vessel. HRP injections in the neonatal animals were made into the inferior vena cava. Five, $15,30,45,60$, or $90 \mathrm{~min}$ after the cessation of the injection, the animals were perfused through the left ventricle with $200 \mathrm{~mL}$ of warm $\left(37^{\circ} \mathrm{C}\right)$ heparinized saline, followed by $500 \mathrm{~mL}$ of $1 \%$ paraformaldehyde $-4 \%$ glutaraldehyde in $0.1 \mathrm{~mol} / \mathrm{L}$ phosphate buffer, $\mathrm{pH} 7.4$, for $15-30$ min. Finally, the fixation was terminated by perfusion of the animal for an additional $15-30 \mathrm{~min}$ with $500 \mathrm{~mL}$ of chilled $\left(4^{\circ} \mathrm{C}\right) 0.1 \mathrm{~mol} / \mathrm{L}$ phosphate buffer containing $10 \%$ sucrose.

Each of the six adult animals in the second group received Class V cavity preparations in the lingual surfaces of the first and second maxillary molar teeth. The preparations were made by use of a low-speed handpiece (Kerr Electrotorque) and a $33 \mathrm{k} / 2$ inverted cone dental bur under continuous saline spray. Each cavity was approximately one millimeter square and 0.75 $\mathrm{mm}$ deep, and extended, at its maximum depth, to within 0.15 $\mathrm{mm}(150 \mu \mathrm{m})$ of the predentin-dentin border. Immediately prior to cavity preparation, the abdominal cavity was opened via a ventral midline incision, and 0.6 to $1.0 \mathrm{~mL}$ of 1 to $2 \%$ HRP in saline was slowly infused with a glass micropipette into the inferior vena cava over a period of ten to $15 \mathrm{~min}$. Fifteen, 30, or 45 min after cavity preparation, successive solutions of saline, fixative, and $10 \%$ sucrose were perfused through the left ventricle, as described in the experiments on normal animals (see above).

Following the perfusion, that portion of the maxilla containing the molar teeth was removed bilaterally and decalcified over a period of two to three weeks in daily changes of icecold, buffered $0.2 \mathrm{~mol} / \mathrm{L}$ sodium ethylenediamine tetra-acetate (EDTA), at $\mathrm{pH} 7.4$. The teeth were then sectioned individually at $30-40 \mu \mathrm{m}$ in a cryostat, and the sections immediately reacted for HRP activity according to either the tetramethylbenzidine (TMB) technique of Mesulam (1978) or the cobalt chloride modification (Adams, 1977) of the diaminobenzidine (DAB, Graham and Karnovsky, 1966) procedure. After completion of the incubations, the sections were washed in three changes of either cold $5 \%$ acetate buffer (TMB procedure) or $0.1 \mathrm{~mol} /$ $\mathrm{L}$ phosphate buffer (DAB procedure). All of the neonatal material and approximately half of the sections from the normal and experimental adult animals were then mounted on chrome alum-gelatin-coated slides, dehydrated, cover-slipped, and ex- amined with an Olympus BH-2 light microscope. The remaining sections were osmicated (Carson and Mesulam, 1982), dehydrated for seven min each (in a series of graded ethanols), and flat-embedded in Spurrs epoxy resin between two Tefloncoated cover slips. Following polymerization, the $80-100-\mu \mathrm{m}$ thick resin wafers were examined under a light microscope, and areas of interest were trimmed from the wafers and glued with quick-setting epoxy to the ends of epoxy resin blocks. Thin $(80-100 \mathrm{~nm})$ sections were cut with a diamond knife, collected on 150-mesh copper grids, and viewed, unstained, in a Phillips 300 electron microscope.

Two types of histochemical controls were performed to verify the specificity of the HRP-tracing procedure used in this study. So that we could test for the presence of endogenous peroxidase activity within the dental pulp, we subjected two adult control animals to vascular injections of $0.9 \% \mathrm{NaCl}$ containing no exogenous HRP. The animals were then perfusionfixed, and the teeth were sectioned and processed, as described above, for the demonstration of HRP activity. To investigate the possibility that some or all of the visualized reaction product may have resulted from the non-specific deposition of incubation media components, we reacted several sections from each experimental animal in an incubation medium lacking either the chromagen (TMB or DAB) or the substrate $\left(\mathrm{H}_{2} \mathrm{O}_{2}\right)$. All the controls proved to be negative.

\section{Results.}

Permeability of the odontoblast cell layer in normal, mature dentition. - Injection of horseradish peroxidase into either the external carotid artery or the inferior vena cava of the adult rats resulted in the rapid and extensive deposition of HRP reaction product throughout all areas of the dental pulp (roots, chamber, and horns). Increasing the survival time following tracer infusion (from 5 to $90 \mathrm{~min}$ ) had no noticeable effect on the distribution of reaction product. Tíssue sections processed according to the TMB or DAB procedures yielded comparable results, although the quality of ultrastructural preservation was slightly better in sections reacted with $\mathrm{DAB}$.

Light microscopic examination of the tissue revealed that the reaction product crystals were distributed relatively uniformly throughout the extracellular compartment of the dental pulp (Figs. 1a,b). Conspicuous deposits of reaction product were present in the periphery of the pulp, immediately beneath the odontoblast cell layer, and within the narrow intercellular spaces between adjacent odontoblasts; however, the predentin and dentin were in every animal devoid of reaction product (Figs. 1c,d).

Electron microscopic (EM) observations confirmed and extended the findings made at the light microscopic level. At the ultrastructural level, the crystals of HRP reaction product were easily distinguishable because of their superior electron density and characteristic crystalline (TMB) or flocculent (DAB) morphology. Reaction product crystals were observed throughout the extracellular spaces of the pulp (Fig. 2a), and in the odontoblast cell layer, they were often observed in the narrow intercellular gaps between adjacent odontoblast cell bodies (Fig. $2 b$ ). However, penetration of reaction product between the apical regions of adjacent odontoblasts was prohibited by areas of membrane fusion that resembled tight junctions (Fig. 2b). No reaction product was observed in the predentin or dentin.

Permeability of the odontoblast cell layer in neonates. The experiments which used neonatal animals provided evidence that functional barriers between developing odontoblasts first appeared between days 15 and 28 of neonatal life. Intraventricular injections of HRP in 3-15-day-old animals resulted 


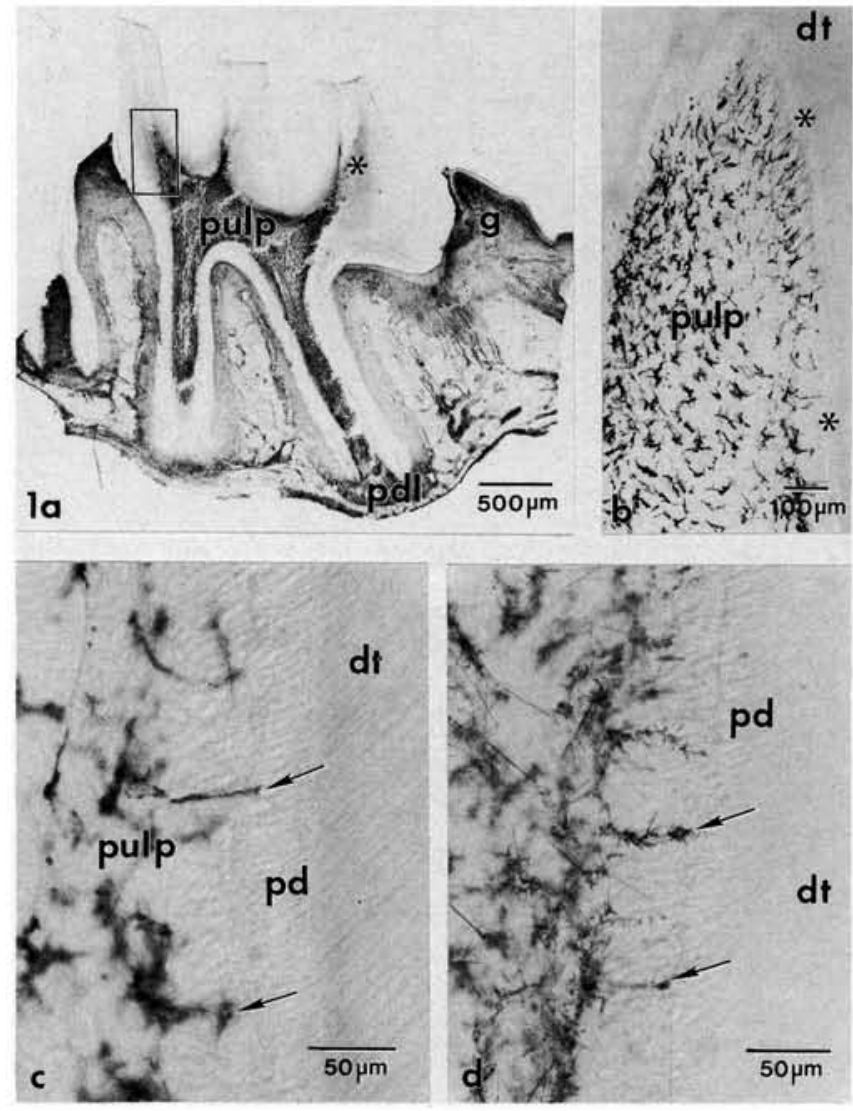

Fig. 1 - Light micrographs showing HRP reaction product in the maxillary molar teeth of normal, adult animals, $15 \mathrm{~min}$ after HRP was injected into the external carotid artery. (a) HRP-TMB reaction product (dark staining material) is distributed heavily and uniformly throughout the dental pulp, periodontal ligament (pdl), and gingiva (g). The slightly darkish area indicated by the asterisk is caused by optical refraction of the dentin and should not be confused with reaction product. An area of pulp horn similar to that enclosed by the box is shown at higher magnification in (b). (b) HRP-DAB reaction product is distributed widely throughout the extracellular spaces of the pulp horn (pulp), but has not entered the predentin (asterisks) or dentin (dt). (c) High-magnification micrograph of the peripheral dental pulp, predentin (pd), and dentin (dt) in a mesial pulp horn. HRP-DAB reaction product is visible in the pulp and between adjacent cells of the odontoblast layer (e.g., arrow), but is not visible distal to the odontoblast/predentin border zonc. (d) Comparable section as in (c) but with TMB as the chromogen.

in widespread deposition of HRP throughout the dental pulp, predentin, and dentin (Fig. 3), whereas injections of animals 28 days and older produced results identical to those seen in the adult, i.e., there was a total absence of reaction product in the predentin and dentin.

Alteration of odontoblast permeability following Class V cavity preparations. - The dental pulps of animals that received Class V cavity preparations after HRP was injected into the blood stream contained deposits of reaction product in the extracellular compartment that were similar in appearance and distribution to those described in the normal adult animals (see above). However, additional quantities of reaction product were also observed in the predentin and dentin immediately beneath the cavity preparation (Fig. 4). Electron microscopic examination of these areas revealed that the HRP reaction product was located in the periodontoblastic spaces surrounding the odontoblast processes (Fig. 5). In contrast, the dentin and predentin in all other areas of the tooth, and in control (nonper-

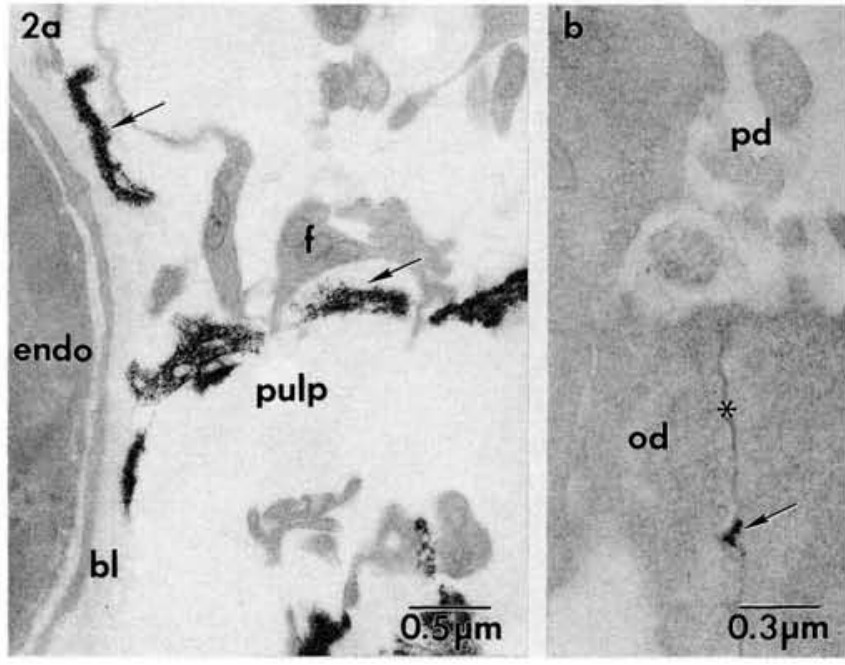

Fig. 2 - Electron micrographs showing HRP reaction product in the dental pulp of normal adult rat molars, $15 \mathrm{~min}$ after tracer was injected into the vascular system. (a) Electron-dense crystals of HRP-DAB reaction product $(e . g$., arrows) are scattered within the extracellular spaces of the pulp. Fibroblast processes (f) and the basal lamina (bl) of a capillary endothelial cell (endo) are also visible. Unstained section. (b) Electron micrograph of the odontoblast-predentin border zone. A crystal of HRP$\mathrm{DAB}$ reaction product (arrow) is visible in the intercellular space between two odontoblasts (od). The odontoblast cell membranes distal to the reaction product are fused in a five-layered complex (asterisk) that suggests a tight junction. Reaction product is absent from the predentin (pd).

turbed) teeth from the same animal, were devoid of reaction product.

\section{Discussion.}

Intravascular injection of the macromolecular tracer, HRP, was used in this investigation to study the permeability of the odontoblast cell layer in developing and mature rat molar teeth, and to examine the effects of cavity preparations on the permeability of this epithelioid cell layer in adult animals. The results have shown that in 3-15-day-old molar teeth, HRP enters the developing dental pulp through the vascular system and penetrates freely between adjacent odontoblasts to enter the predentin and dentin. In contrast, in animals aged 28 days and older, HRP enters the pulp and passes between adjacent odontoblasts as far as the pulp-predentin border, but no further. When Class V cavity preparations were performed on adult teeth immediately after HRP infusion, the tracer penetrated the predentin and dentin subjacent to the base of the cavity; however, remaining (nontraumatized) areas of dentin and predentin remained devoid of reaction product. We conclude from these investigations that: (1) a functional barrier develops between the distal segments of pulpal odontoblastic cell bodies in normal rat molar teeth between days 15 and 28 of postnatal life, and these barriers prevent the passage of macromolecules from the pulp into predentin and dentin, and (2) this barrier is perturbed following routine restorative procedures.

Technical considerations. - In this study, the light and electron microscopic visualization of HRP was accomplished with use of both $\mathrm{DAB}$ and TMB histochemical procedures. The chromagen $\mathrm{DAB}$ possesses the advantage of forming a discrete reaction product which, at the ultrastructural level, is confined in its distribution to the subcellular compartment(s) involved in the uptake or transport of the HRP (Graham and Karnovsky, 1966). The chromagen TMB, in contrast, gives 
3a

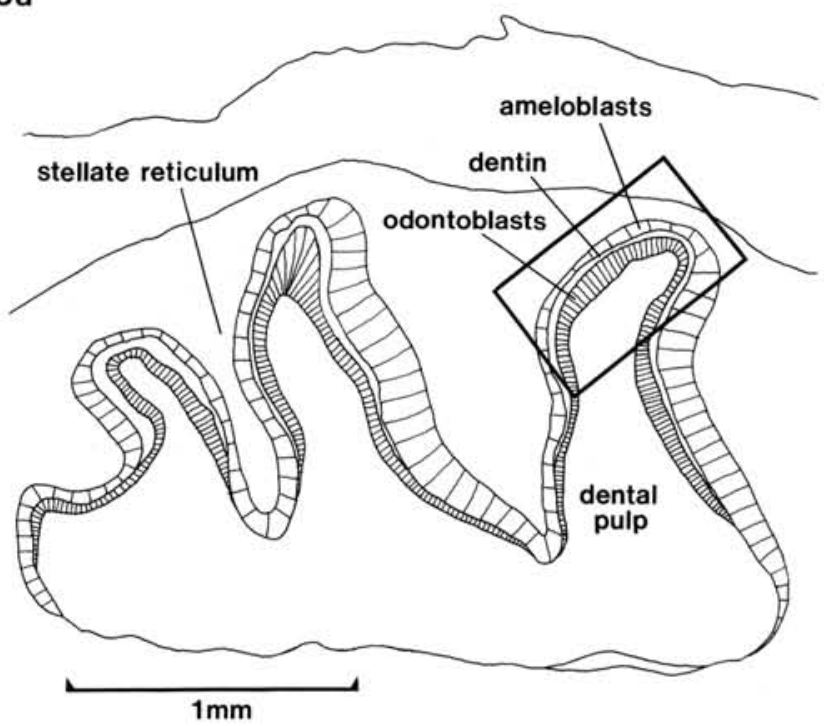

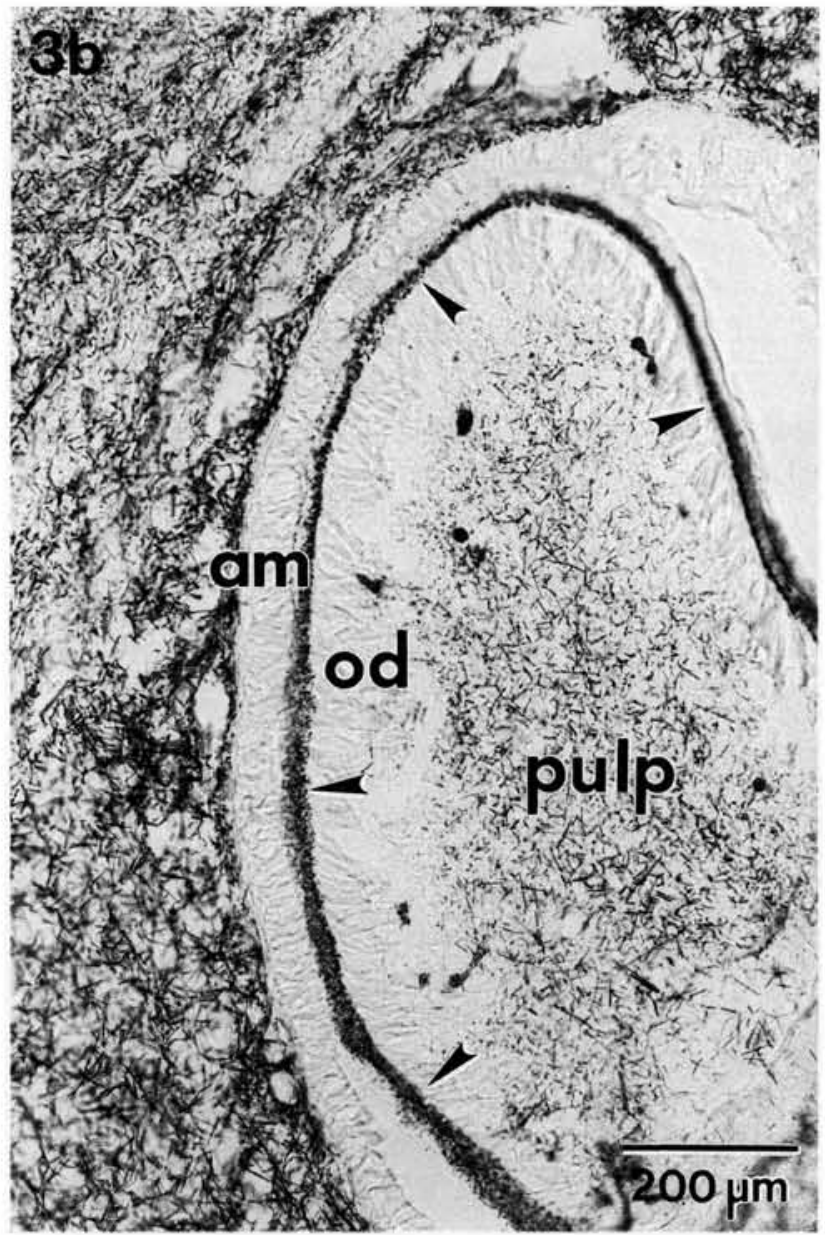

Fig. 3 - Distribution of HRP-TMB reaction product in the first maxillary molar tooth bud of a 3-day-old rat. The tooth bud is in the bell (dentinogenesis) stage of tooth development and is characterized by the presence of an ameloblast (am) layer, an odontoblast (od) layer, and a thin, intervening layer of predentin and dentin. The ameloblasts have not yet begun to form enamel. The area of tooth bud illustrated in the right figure ( $3 \mathrm{~b}$ ) is outlined by the box in the orientation diagram (3a). A dense band of HRP-TMB reaction product is located in the predentin/dentin (arrowheads). The tracer most likely reached the dentin by passing through the odontoblast cell layer (as opposed to entering via the ameloblast layer), since at higher magnification numerous granules of reaction product were observed in the intercellular spaces between odontoblasts, but only rarely were they seen between ameloblasts.

rise to a reaction product that usually exceeds in size the distribution of the exogenous HRP (Carson and Mesulam, 1982). However, the use of TMB offers two distinct advantages that made it particularly attractive for use in this study: (1) It is generally felt that, at both the light and electron microscopic levels, TMB affords a level of sensitivity considerably higher than that of DAB (Mesulam, 1978; Carson and Mesulam, 1982); and (2) the crystalline substructure of the TMB reaction product at the ultrastructural level is unique, which greatly facilitates its differentiation from endogenous tissue components (Sakumoto et al., 1980; Marfurt et al., 1988). The results of this study and of earlier work in our laboratories (Marfurt and Turner, 1983; Marfurt et al., 1988) have shown that the HRP enzyme will survive the decalcification procedure used in this study, in quantities sufficient to catalyze the oxidation of a suitable chromagen, i.e., TMB or DAB. Nevertheless, we acknowledge that some loss of enzyme activity may have occurred following decalcification. Therefore, small amounts of HRP could have diffused into the predentin space and gone undetected by the less-sensitive DAB protocol. However, we believe that, by using TMB and taking advantage of the "phenomenon of histochemical magnification" (Carson and Me- sulam, 1982), even small amounts of the enzyme can be localized and identified.

The nature of the barrier function: a role for junctional complexes? - Specialized areas of membrane contact between odontoblasts have been known since the initial electron microscopic studies of Frank (1968) and his contemporaries (Jessen, 1967; Arwill, 1967, 1968; Garant et al., 1968; Reith, 1968). These early observations have subsequently been confirmed and extended by a number of different workers who used both conventional transmission electron microscopy (Takuma and Nagai, 1971; Holland, 1975; Sasaki et al., 1982a; Bishop, 1987) and freeze-fracture techniques (Sasaki et al., 1982a,b; Iguchi et al., 1984; Calle, 1985), and as a result, three basic types of odontoblastic junctional complexes are now widely recognized: desmosomes, gap junctions, and tight junctions. Desmosomes and gap junctions are numerous along the lateral and basal aspects of the cells. The former junctional contacts are important in cell-to-cell adhesion and in maintaining the polarity and epithelioid nature of the odontoblast cell layer. Gap junctions represent between-cell areas of low electrical resistance through which ions and metabolites move freely, and although their precise function in odontoblast physiology 


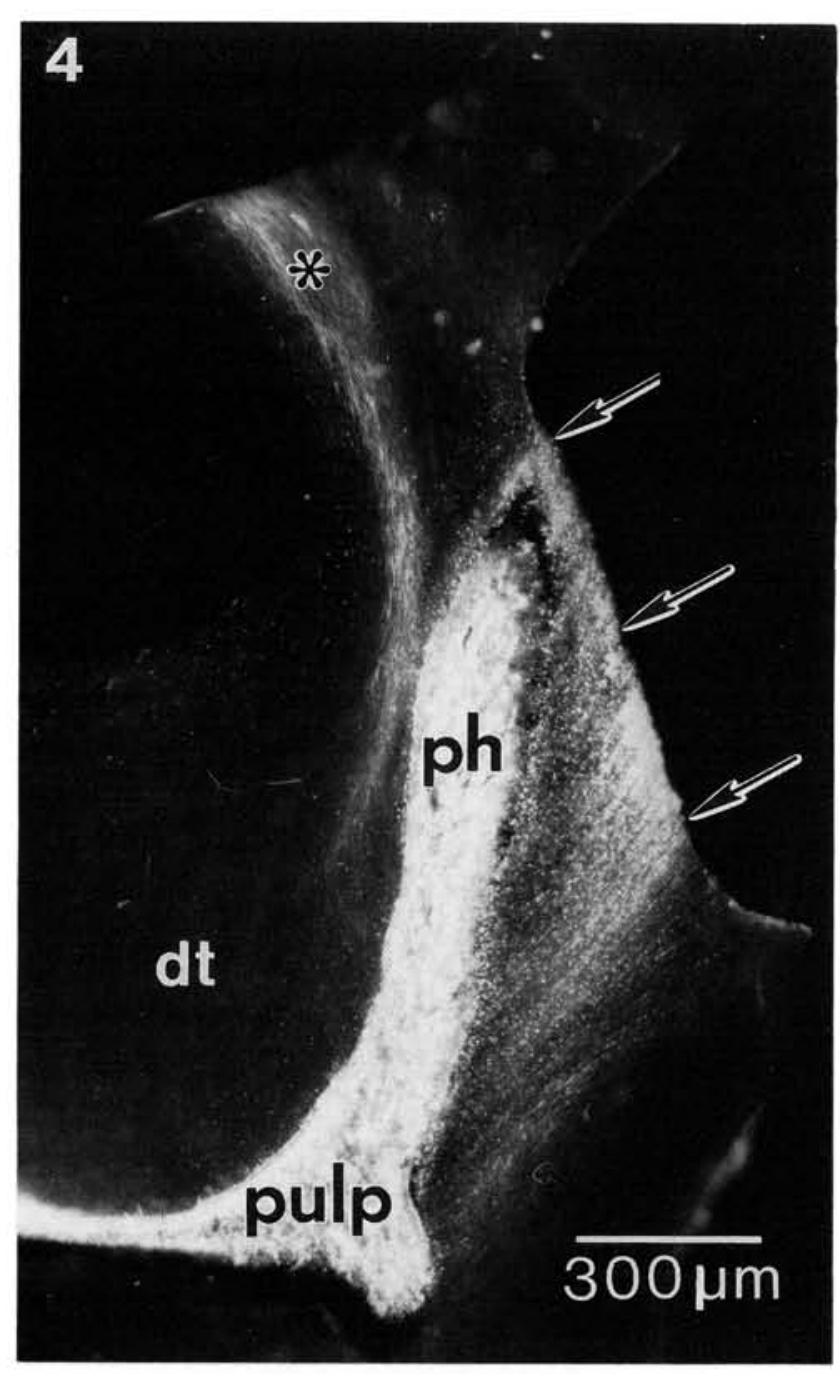

Fig. 4 - Darkfield light micrograph of a pulp horn from a molar tooth that received a Class $V$ cavity preparation after HRP injection into the blood stream. HRP-TMB reaction product fills the pulp chamber (pulp) and pulp horn (ph), and extends into the dentin subjacent to the base of the cavity preparation (arrows). The surrounding, unperturbed areas of dentin (dt) are devoid of reaction product. The whitish area indicated by the asterisk is a region of refractile dentin and is not to be confused with reaction product.

remains speculative, it has been postulated (Sasaki et al., 1982b) that they may play important roles in the cytodifferentiation of odontoblasts and in the synchronization of odontoblast function, especially with regard to the elaboration and mineralization of dentin. However, because desmosomes and gap junctions are distributed in spotlike fashion, and because narrow intercellular spaces exist between the outer lamellae of the adjacent plasma membranes, it is unlikely that they contribute in any appreciable fashion to the odontoblastic barrier function. $\AA$ more likely candidate for the barrier function seen in the current investigation is the tight junction, where the adjacent outer lamellae of apical odontoblast cell membranes appear to fuse and, therefore, obliterate the intercellular space for a variable distance. Tracer studies in other epithelia have shown that HRP or lanthanum placed in the tissue space cannot cross the tight junctions to reach the free surface of the tissue (e.g., Goodenough and Revel, 1970). In the odontoblast cell layer, some authors claim that odontoblast tight junctions form continuous rings (or belts) of membrane fusion about the circumference of the cell apex (i.e., "true" tight junctions, on zonula occludens-Frank, 1966; Reith, 1968; Calle, 1985); however, others feel that they are arranged in a series of focal cellular fusions ("macular" tight junctions) analogous to "spot rivets" (Garant et al., 1968; Sasaki et al., 1982; Iguchi et al., 1984). The resolution of this apparent controversy is perhaps beyond the capabilities of conventional morphological techniques, because these methods reveal only very small areas of cell membrane in a given section; thus, it is difficult to ascertain by means of this procedure, to what extent the intercellular junctions encircle the cells in three dimensions.

In this study, therefore, we have investigated the nature of the odontoblast permeability barrier in molar teeth of normal adult and developing animals, by using a more three-dimensional approach, i.e., by introducing the exogenous tracer, HRP, into the dental pulp, and monitoring its passage through the odontoblast cell layer and into the predentin and dentin. The results of earlier studies of this type, which used the same or different tracer substances, resulted in the publication of what appears to be yet another collection of contradictory reports (Tanaka, 1980; Sasaki et al., 1982b; Bishop, 1985). However, the results of our study strongly suggest that the differences in odontoblast permeability reported by other investigators may be largely explained by variation in the developmental stage of the tooth examined. Thus, we propose that the odontoblast cell layer is permeable to horseradish peroxidase and lanthanum nitrate in developing, pre-eruptive-stage teeth (Sasaki et al., 1982; Tanaka, 1980; current investigation), but that it is impermeable to the passage of these same compounds in adult, fully developed teeth (Bishop, 1985; current investigation). Furthermore, we hypothesize that the appearance of this barrier may be related to the development of zonular tight junctions between the apical regions of the odontoblasts. This theory receives support from electron microscopic and freeze-fracture studies: Macular (discontinuous) tight junctions have been observed between odontoblasts of developing teeth (Sasaki et al., 1982b; Iguchi et al., 1984), whereas zonular tight junctions are seen between the apical regions of the cells in mature teeth (Calle, 1985). It is not known whether the macular tight junctions observed between young odontoblasts enlarge and fuse to form zonular tight junctions between mature odontoblasts. However, assemblage (or "maturation") of zonulae occludens from multiple focal tight junctions has been reported in other epithelial tissues (Tice et al., 1977; Dermietzel et al., 1977).

Functional considerations. - The appearance of zonulae occludens in mature dental pulp likely indicates a shift in function, or the addition of new functions, for the odontoblasts. Macular tight junctions between odontoblasts of immature teeth are probably important for cell-to-cell attachment (Sasaki et al., 1982b), a role consistent with the proposed function of tight junctions in other types of epithelia (Grynszpan-Wynograd and Nicolas, 1980). Indeed, tight junctions show considerable strength and are the last elements of the junctional complex to break under tension (Farquhar and Palade, 1963). However, it is unlikely that macular tight junctions, because of their discontinuous nature, are able to regulate fluid and ion movements between the pulp and dentin. Thus, during dentinogenesis, minerals, metabolites, and other components of dentinal fluid can pass freely through the odontoblast layer by both extracellular (Sasaki et al., 1982a; Tanaka, 1980) and intracellular (Reith, 1968; Nagai and Frank, 1974) routes to reach the mineralization front. In contrast, zonulae occludens between mature, secretory odontoblasts form continuous, beltlike barriers between the pulp and predentin. Functionally, zonular tight junctions may partially regulate fluid movement 
Fig. 5 - Electron micrograph illustrating crystals of HRP-TMB reaction product (arrows) surrounding odontoblast processes (op) in the predentin (pd) beneath a cavity preparation. Od, odontoblast.

through dentinal tubules. Fluid movement through dentin is thought to be regulated or "resisted"' by a combination of three factors: surface resistance, intratubular resistance, and pulpal resistance (Pashley et al., 1978, 1981). The tight junctions demonstrated experimentally in this study may well be the source of the "pulpal" resistance. Zonulae occludens also function to maintain chemical and electrochemical gradients across the odontoblast cell layer, by impeding the back-diffusion (leakage) of substances along the intercellular spaces between odontoblasts. Thus, in fully formed teeth, the passage of fluid, ions, and other molecules along the extracellular pathway is hindered, and transfer of these substances from pulp to dentin must occur largely through intra-odontoblastic, transcellular mechanisms. The composition of dentinal fluid in the adult, therefore, is apparently highly regulated and is not simply a transudate of pulpal capillaries (Bishop, 1987).

Odontoblasts may also play a role in dentin sensitivity by regu-

lating the ionic composition of the dentinal fluid that surrounds intradentinal nerve fibers. However, the importance of the odontoblast cell layer in this regard is uncertain, since pain can be evoked from dentin with no odontoblasts beneath it (Brännström and Åström, 1964), and nerves can continue to be activated by dentinal stimulation despite damage to the peripheral pulp and odontoblasts (Hirvonen and Närhi, 1986). Interestingly enough, the impermeability of the adult odontoblast cell layer to HRP and lanthanum (Bishop, 1985; current investigation) is maintained even in the pulp horns, where sensory fibers pass between adjacent odontoblasts to enter the dentinal tubules (see Byers, 1984, for review). The mechanism responsible for maintaining the barrier to exogenous substances between these disparate cell types is presently unknown. Although gap junctions between odontoblasts and "nerve-like", processes have been reported by some workers (Holland, 1975, 1976; Matthews and Holland, 1975; Koling and Rask-Andersen, 1984a), more recent anatomical (Byers, 1977, 1979; Holland, 1980; Turner, 1982) and experimental denervation (Holland, 1987) studies have been unable to confirm these observations.

Breakdown of zonulae occludens in the odontoblast layer following Class $V$ cavity preparations. - The results of the present experimental tracer study have demonstrated that the functional barrier to HRP passage that exists between pulp and dentin in normal adult teeth is perturbed following routine restorative procedures. We hypothesize that the loss of the odontoblast barrier function is probably caused, at least in part, by the disruption of the zonulae occludens that normally link these cells together. The short-term physiological and metabolic responses of the odontoblasts to cavity preparation and severance of the distal aspect of the odontoblast process are unknown. However, the present results suggest that, when perturbed by a Class V cavity preparation, zonulae occludens between odontoblasts rapidly dissolve (or re-arrange to become "macular"), as evidenced by the immediate, prominent, and highly localized passage of HRP from the pulp into the peritubular spaces of the overlying dentin. Functionally, this phenomenon of enhanced permeability may indicate an attempt by the odontoblast cell layer to increase the rate of transfer of reparative compounds and associated ions into the overlying predentin, as a first step toward repairing the damage to the overlying tooth matrix.

The elegant in vivo studies of Pashley et al. (1983a,b, 1984) are consistent with the observations of this study. The latter workers have shown that dentin permeability is high immediately following routine cavity preparations, then falls progressively to a level of about $20 \%$ of zero-time values in five to six h. Considering the data presented here, these observations could be explained by the perturbation of zonulae occludens following routine cavity preparation, and the subsequent restoration of this barrier with time. This hypothesis raises broader questions concerning the dynamic properties of odontoblast tight junctions under normal and pathological conditions, and additional studies are needed to learn to what extent odontoblast tight junctions can assemble, disassemble, and re-assemble in response to functional demand. 


\section{Acknowledgments.}

We are grateful to Mark Jones for expert technical assistance and to Connie Lopatin for preparation of the manuscript.

\section{REFERENCES}

ADAMS, J.C. (1977): Technical Considcrations on the Use of Horseradish Peroxidase as a Ncuronal Marker, Neuroscience 2:141-145.

ARWILL, T. (1967): Studies on the Ultrastructure of Dental Tissues, Odont Revy 18:191-208.

ARWILL, T. (1968): The Ultrastructure of the Pulpo-Dentinal Border Zonc. In: Dentin and Pulp, N.B.B. Symons, Ed., Baltimore: Williams and Wilkins Co., pp. 147-167.

BISHOP, M.A. (1985): Evidence for Tight Junctions Between Odontoblasts in the Rat Incisor, Cell Tissue Res 239:137-140.

BISHOP, M.A. (1987): An Investigation of Pulp Capillaries and Tight Junctions Between Odontoblasts in Cats, Anat Embryol 177:131-138.

BRANNSTROM, M. (1963): Dentin Sensitivity and Aspiration of Odontoblasts, J Ami Dent Assoc 66:366-370.

BRÄNNSTROM, M. and ÅSTRÖM, A. (1964): A Study on the Mechanism of Pain Elicited from the Dentin, $J$ Dent Res 43:619-625.

BYERS, M.R. (1977): Fine Structure of Trigeminal Receptors in Rat Teeth. In: Pain in the Trigeminal Region, D.J. Anderson and B. Matthows, Eds., North Holland: Elscvicr, pp. 13-24.

BYERS, M.R. (1979): Large and Small Trigeminal Nerve Endings and Their Associations With Odontoblasts in Rat Molar Dentin and Pulp, Ads: Pain Res Ther 3:265-270.

BYERS, M.R. (1984): Dental Scnsory Receptors, Int Rev Netrobiol 25:3993.

CARSON, K.A. and MESULAM, M.-M. (1982): Electron Microscopic Demonstration of Noural Connections Using Horscradish Pcroxidasc: a Comparison of the Tetramethylbenzidine Procedure with Seven Other Histochemical Mcthods, I Histochem Cytochem 30:425-435.

CALLE, H. (1985): Intercellular Junctions Between Human Odontoblasts, Acta Anat 122:138-144.

DERMIETZEL, R.; MELLER, K.; TETZLAFF, W.; and WAELSH, M. (1977): In vivo and in vitro Formation of the Junctional Complex in Choroid Epithelium. A Frecze-etching Study, Cell Tissue Res 181:427$44 !$.

EDA, S. and SAITO, T. (1978): Electron Microscopy of Cells Displaced into the Dentinal Tubules duc to Dry Cavity Prcparation, I Oral Pathol $7: 326-335$

FARQUHAR, M.G. and PALADE, G.E. (1963): Junctional Complexes in Various Epithclia, $J$ Cell Biol 17:375-408

FRANK, R.M. (1968): Ultrastructural Relationship Between the Odontoblast, its Process and the Nerve Fibre. In: Dentine and Pulp. Their Structure and Reactions, N.B.B. Symons, Ed., Baltimorc: Williams and Wilkins Co., pp. 115-146.

GARANT, P.R.; SZABO, G.; and NALBANDIAN, J. (1968): The Fine Structure of the Mouse Odontoblase, Anch Oral Biol 13:857-876.

GRAHAM, R.C. and KARNOVSKY, M.J. (1966): The Early Stages of Ahsorption of Injected Horseradish Peroxidase in the Proximal Tubule of Mouse Kidney: Ultrastructural Cytochemistry by a New Technique, I Histochem Cytochem 14:291-302.

GOODENOUGH, D.A. and REVEL, J.P. (1970): A Finc Structural Analysis of Intercellular Junctions in the Mousc Liver, $J$ Cell Bied $45: 272-290$

GRYNSZPAN-WYNOGRAD, O. and NICOLAS, G. (1980): Intercellular Junctions in the Adrenal Medulla: A Comparative Frecze Fracture Study, Tissuc Cell 12:661-672.

HIRVONEN, T.J. and NÄRHI, M. (1986): The Effect of Dentinal Stimulation on Pulp Nerve Function and Pulp Morphology in the Dog, $J$ Dent Res 65:1290-1293.

HOLLAND, G.R. (1975): Membranc Junctions on Cat Odontoblasts. Arch Oral Biol 20:551-55?

HOLLAND, G.R. (1976): Lanthanum Hydroxide Labcling of Gap Junctions in the Odontoblast Layer, Anat Rec 186:121-126.

HOLLAND, G.R. (1980): Non-myclinated Nerve Fibers and Their Terminals in the Subodontoblast Plcxus of the Feline Dental Pulp, I Antat 130:457-467.

HOLLAND, G.R. (1987): The Effect of Nerve Section on the Incidenee and Distribution of Gap Junctions in the Odontoblast Layer of the Cat, Anat Rec 218:458-465.

IGUCHI, Y.; YAMAMURA, T.; ICHIKAWA, T.; HASHIMOTO, S.; HORIUCHI, T.; and SHIMONO, M. (1984): Intercellular Junctions in Odontoblasts of the Rat Incisor Studied with Frecze-Fracture, Arch Oral Biol 29:487-497.
IESSEN, H. (1967): The Ultrastructure of Odontoblasts in Perfusion Fixed, Demineralized Incisors of Adult Rats, Acta Odontol Scand 25:491523

JOHNSON, G.; OLGART, S.; and BRÄNNSTRÖM, M. (1973): Outward Fluid Flow in Dentin Under a Physiologic Pressure Gradient: Experiments in vitro, Oral Surg Oral Med Oral Pathol 35:238-248.

KOLING, A.; RASK-ANDERSEN, $\mathrm{H}$; and BAGGER-SJOBACK, D (1981): Membranc Junctions on Odontoblasts. A Freczc-Fracture Study, Acta Odontol Scand 39:355-360.

KOLING, A. and RASK-ANDERSEN, H. (1984a): Mcmbrane Junctions Between Odontoblasts and Associated Cells. A Freeze-Fracture Study of the Human Odontoblastic Cell Layer with Special Reference to its Nerve Supply, Acta Odontol Scand 42:13-22.

KOLING, A. and RASK-ANDERSEN, H. (1984b): Membrane Structures in the Pulp Dentin Border Zone. A Frecze-fracture Study of Demineralized Human Teeth, Acta Odontol Scand 138:355-360.

MARFURT, C.F. and TURNER, D.F. (1983): Sensory Nerve Endings in the Rat Orofacial Region Labcled by the Antcrograde and Transganglionic Transport of Horseradish Peroxidase: a New Method for Tracing Peripheral Nerve Fibers, Brain Res 261:106-117.

MARFURT, C.F.; TURNER, D.F.; and ADAMS, C.E. (1988): Stabilization of Tetramethylbenzidine (TMB) Reaction Product at the Electron Microscopic Level by Ammonium Molybdate, I Neurosci Meths $25: 215-223$

MATTHEWS, B. and HOLLAND, G.R. (1975): Coupling Between Nerves in Tecth, Brain Res 98:354-358

MESULAM, M.M. (1978): Tetramethyl Benzidine for Horscradish Pcroxidasc Ncurohistochemistry: a Non-carcinogenic Bluc Reaction Product with Superior Sensitivity for Visualizing Neural Afferents and Efferents, $J$ Histochem Cytochem 26:106-117.

NAGAI, N. and FRANK, R.M. (1974): Electron Microscopic Autoradiography of Cats During Dentinogenesis, Cell Tissue Res 155:513523

PASHLEY, D.H.; KEPLER, E.E.; WILLIAMS, E.C.; and OKABE, A. (1983a): The Effects of Acid Etching on the in wive Permcability of Dentinc in the Dog, Arch Oral Biol 29:853-858.

PASHLEY, D.H.; KEPLER, E.E.; WILLIAMS, E.C.; and OKABE, A. (1983b): Progressive Decrease in Dentinc Permeability Following Cavity Preparation, Arch Oral Biol 28:853-858.

PASHLEY, D.H.; KEPLER, E.E.; WILLIAMS, E.C.; and O'MEARA, J.A. (1984): The Effect on Dentine Permeability of Time Following Cavity Preparation in Dogs, Arch Oral Biol 29:65-68.

PASHLEY, D.H.; LIVINGSTON, M.J.; and GREENHILL, J.D. (1978): Regional Resistance to Fluid Flow in Human Dentine in vitro, Arch Oral Biol 23:807-810.

PASHLEY, D.H.; NELSON, R.; and WILLIAMS, E.C. (1981): Dentinc Hydralulic Conductance: Changes Produced by Red Blood Cells, $J$ Dem Res 60:1779-1802.

REITH, E.J. (1968): Collagen Formation in Developing Molar Tecth of Rats, J Ultrastruct Rex 21:383-414.

SAKUMOTO, T.; NAGAI, T.; KIMURA, H.; and MAEDA, T. (1980): Electron Microscopic Visualization of Tetramethyl-Benzidinc Reaction Product on Horscradish Peroxidase Neurohistochemistry, Cell Mol Biol $26: 211-216$

SASAKI, T.; ISHIDA, I.; and HIGASHI, S. (1982a): Uttrastructure and Cytochemistry of Old Odontoblasts in Rat Incisors, $J$ Electron Microsc $31: 378-388$

SASAKI, T.; NAKAGAWA, K.; and HIGASHI, S. (1982b): Ultrastructure of Odontoblasts in Kitten Tooth Germs as Revaled by Freczefracture, Arch Oral Biol 27:897-904.

SATTELBERG, C.F. and TURNER, D.F. (1984): Anatomical Evidence for the Existence of Zomula Occludens Between Pulpal Odontoblasts, $J$ Dent Res 63:225, Abst. No. 494.

SATTELBERG, C. and TURNER, D.F. (1985): Permeathility of the Pulpal Odontoblast Cell Laycr Following Cavity Prcparation, $J$ Dent Res 64:240, Abst. No. 588

STEWART, J.M. (1965): The Immediate Response of Odontoblasts to Injury, Odon Tidskr 73:417-423.

TAKUMA, S. and NAGAI, N. (1971): Ultrastructure of Rat Odontoblasts in Various Stages of Their Development and Maturation, Arch Oral Biol 16:993-1011.

TANAKA, T. (1980): The Origin and Localization of Dental Fluid in Developing Rat Molar Tecth Studied With Lantlianum as a Tracer, Arch Oral Biol 25:153-162.

TICE, L.W.; CARTER, R.L.; and CAHIL, M.C. (1977): Tracer and Frecze-fracture Observations on Developing Tight Junctions in Fetal Rat Thyroid, Tissue Cell 9:395-418.

TURNER, D.F. (1982): Ultrastructure of the Primatc Dental Pulp Organ, Anat Rec 202:193A. 